\title{
EFEITO DA VOLATILIDADE DA TAXA DE CÂMBIO NAS EXPORTAÇÕES BRASILEIRAS
}

\author{
EFFECTS OF EXCHANGE RATE VOLATILITY ON BRAZILIAN \\ EXPORTS
}

\section{EFECTOS DE LA VOLATILIDAD DEL CAMBIO EN LAS EXPORTACIONES BRASILENÃS}

\author{
Osmar José Bertholini Pianca \\ Mestre em Administração, na linha de pesquisa Finanças \\ e Avaliação de Empresas / Professor do Instituto Federal \\ do Espírito Santo (IFES), Campus Linhares, Brasil \\ osmarpianca@hotmail.com
}

\section{Carlos Heitor Campani}

PhD em Finanças pela Edhec Business School, França / Professor de Finanças do Instituto de Pós-Graduação e Pesquisa em Administração (COPPEAD) da Universidade Federal do Rio de Janeiro (UFRJ), Brasil / Pesquisador associado do Instituto Edhec-Risk, França carlos.heitor@coppead.ufrj.br

\author{
Rafael Cardoso do Nascimento \\ Pesquisador do Instituto de Pós-Graduação e Pesquisa em \\ Administração (COPPEAD) da Universidade Federal do \\ Rio de Janeiro (UFRJ), Brasil \\ rafael.cardoso@coppead.ufrj.br
}

\author{
Contextus \\ ISSNe 2178-9258 \\ Organização: Comitê Científico Interinstitucional \\ Editor Científico: Carlos Adriano Santos Gomes \\ Avaliação: double blind review pelo SEER/OJS \\ Edição de texto e de layout: Carlos Daniel Andrade \\ Recebido em 25/07/2017 \\ Aceito em 23/10/2017 \\ $2^{a}$ versão aceita em $06 / 11 / 2017$
}

\section{RESUMO}

Uma economia com alta volatilidade no câmbio gera incertezas em seu fluxo de comércio internacional, o que acaba por impactar suas exportações. Tais incertezas ensejam pesquisas que procuram identificar os efeitos da volatilidade da taxa de câmbio sobre as exportações dos países. O presente trabalho visa investigar o impacto da volatilidade do câmbio de dólar estadunidense nas exportações do Brasil com foco em certos setores relevantes da economia brasileira. Assim, tomam-se como variáveis independentes quatro métricas distintas de volatilidade cambial e, como variáveis dependentes, os volumes exportados de alguns produtos e setores. As estimativas foram realizadas pelo Método Generalizado dos Momentos, por este apresentar melhores propriedades para amostras finitas e tratar problemas de endogenia. Os resultados demonstram que alguns produtos e setores são afetados pela volatilidade da taxa de câmbio do dólar estadunidense de forma negativa, enquanto outros produtos ou setores parecem não sofrer efeito algum.

Palavras-chave: Volatilidade cambial. Taxa de câmbio real. Taxa de câmbio nominal. Exportações brasileiras. Brasil.

\begin{abstract}
An economy with high exchange rate volatility brings uncertainties to its international trade flow and, therefore, affects its country's exports. Such uncertainties justify researches trying to identify the effects of exchange rate volatility on exports. This paper aims to investigate the effects of the US dollar exchange rate volatility on Brazilian exports with a focus on certain relevant sectors of Brazil's economy. Four different metrics of exchange rate volatility were used as independent variables whereas the exported volumes of some products and sectors were taken as dependent variables. The estimation process was carried out by means of the Generalized Method of Moments because it presents better properties for finite samples and it treats the endogenicity problem. The results show that some products and sectors are indeed affected by volatility of the US dollar exchange rate in a negative way, while other products and sectors seem to have not been affected.
\end{abstract}

Keywords: Exchange rate volatility. Real exchange rates. Nominal exchange rates. Brazilian Exports. Brazil. 


\section{RESUMEN}

Una economía con alta volatilidad em su cambio genera incertidumbres a su flujo de comercio internacional, lo que acaba por impactar sus exportaciones. Debido a tales incertidumbres, hay investigaciones que buscan identificar los efectos de la volatilidad de cambio en las exportaciones de los países. El presente trabajo busca investigar los efectos de la volatilidad del cambio del dólar estadounidense en las exportaciones de Brasil en ciertos sectores relevantes de la economía brasileña. Así, se tomó como variables independientes cuatro métricas diferentes de la volatilidad cambiaria y, como variables dependientes, los volúmenes exportados de algunos productos y sectores. Las estimaciones fueron realizadas con el Método Generalizado de los Momentos, porque este presenta mejores propiedades para muestras finitas y trata problemas de endogenía. Los resultados demuestran que algunos productos y sectores se ven afectados por la volatilidad del cambio del dólar estadounidense de forma negativa, mientras que otros productos y sectores parecen no sentir ningún efecto.

Palabras clave: Volatilidad cambiaria. Tipo de cambio real. Tipo de cambio nominal. Exportaciones brasileñas. Brasil.

\section{INTRODUÇÃO}

O regime de câmbio flutuante é assunto bastante discutido no contexto do comércio internacional, pois - conforme indicam Bakhromov (2011) e Baum e Caglayan (2010) - a volatilidade da taxa de câmbio pode ter maior ou menor impacto no comércio entre países. Desde o colapso do acordo de Bretton Woods, houve uma transformação gradativa no regime cambial dos países: muitos abandonaram o câmbio fixo e adotaram o flutuante (TAKAENDA; TSHEOLE; AZIAKPONO, 2006). Arize, Osang e Slottje (2008) também destacam a relação entre aquele colapso e a volatilidade cambial.

Desse modo, as exportações dos países podem sofrer flutuações perante seus parceiros comerciais, porém ainda não há consenso sobre o caráter positivo ou negativo de tal efeito (VITA; ABBOTT, 2004). Enquanto alguns estudos encontraram um impacto negativo da volatilidade da taxa de câmbio sobre as exportações (ARIZE; OSANG; SLOTTJE, 2008; CHOWDHURY, 1993), outros identificaram impacto positivo (ALTINTAŞ; CETIN; ÖZ, 2011; SERENIS; TSOUNIS, 2014). Existem ainda pesquisas que não encontraram efeitos significativos (ARISTOTELOUS, 2001; WESSEH; NIU, 2012) e aquelas que apresentaram efeitos mesclados (WANG; BARRETT, 2007; ZHANG; BUONGIORNO, 2010). O presente estudo visa contribuir para o debate analisando o caso brasileiro.

Conforme destacam Caglayan e Di (2010), os resultados da volatilidade são conflitantes e sensíveis a vários fatores. Ozturk (2009) corrobora isso ao constatar que a volatilidade da taxa de câmbio tem sobre o comércio efeitos sensíveis: à escolha do período da amostra, aos modelos de especificação, à escolha das medidas de volatilidade e ao grau de desenvolvimento dos países em análise. 
Conforme destacam Osang, Slottje e Arize (2000), Aziakpono, Takaenda e Tsheole (2006), Bakhromov (2011) e Vergil (2002), pouco se conhece em países menos desenvolvidos devido à falta de dados ou à baixa qualidade destes. Esta pesquisa buscou preencher um pouco tal lacuna, identificando efeitos da volatilidade da taxa de câmbio do dólar estadunidense nas exportações em diversos setores e produtos relevantes da economia brasileira. Esse ponto é inovador, na medida em que busca trazer um poder informacional muito maior do que uma análise agregada das exportações brasileiras, como feito em estudos anteriores. Foram utilizados dados originais e atualizados, bem como se expandiu a análise para as taxas de câmbio real e nominal, ao passo que estudos anteriores tenderam a usar somente a taxa de câmbio real, em maior escala, ou somente a taxa nominal.

O Brasil é o país escolhido para o estudo, pois, desde 1999, possui regime de câmbio flutuante. A propósito, a mudança no regime brasileiro de câmbio fixo para flutuante elevou fortemente, segundo Teles (2005), a variabilidade da taxa cambial e, como consequência, o risco de entrada no mercado externo. Além dessa razão, a importância e o tamanho da economia brasileira no cenário internacional trazem natural relevância para esta pesquisa.
O restante do trabalho está estruturado da seguinte forma. A próxima seção apresenta uma breve discussão da literatura. A terceira explica a metodologia utilizada e fornece uma descrição dos dados utilizados. A quarta apresenta os resultados e os discute. Na última seção, expõem-se algumas considerações finais.

\section{2 \\ DISCUSSÃO DA LITERATURA}

\subsection{Efeitos da volatilidade da taxa de câmbio no fluxo de comércio dos países}

Apesar da opinião generalizada de que as mudanças na volatilidade das variáveis financeiras têm impactos significativos sobre o fluxo de comércio, a evidência empírica é notoriamente mista. Uma série de modelos teóricos, bem como estudos empíricos de diferentes grupos de países têm sido propostos para explicar o impacto da volatilidade da taxa de câmbio sobre as exportações dos países. Não obstante, os resultados ainda se mostram heterogêneos.

Osang, Slottje e Arize (2000) encontraram efeitos negativos da volatilidade sobre as exportações, com uma volatilidade maior surgindo como custo adicional para os comerciantes, que normalmente possuem grande aversão ao risco. O descasamento entre a taxa de câmbio (na maioria das vezes acordada no 
contrato) e o pagamento (na maioria das vezes feito na entrega física ou mesmo depois) expõe o exportador ao risco da flutuação, devendo ele precificar a fonte de risco adequadamente, o que gera um custo adicional ao produto.

Por outro lado, há também estudos apontando efeitos positivos da volatilidade da taxa de câmbio no fluxo de comércio dos países, como por exemplo, Serenis e Tsounis (2014). Por sua vez, Aristotelous (2001) observou ausência de impacto da volatilidade da taxa de câmbio nas exportações britânicas para os Estados Unidos. Como se pode notar, a literatura é bastante controversa, e o efeito parece depender de outros fatores.

Byrne, Darby e MacDonald (2008) encontraram diferentes resultados entre as indústrias: efeito negativo e significativo para produtos diferenciados e efeito insignificativo para produtos homogêneos. Tal disparidade sugere, portanto, que os efeitos podem variar entre setores e que outras variáveis podem influenciar a relação entre volatilidade cambial e exportações, tais como o nível de competição, a natureza do contrato, os instrumentos existentes de hedge e o nível de abertura ao comércio internacional, dentre outros.

Hsu e Chiang (2011), de forma interessante, observaram que o efeito da volatilidade no volume de exportações dos
Estados Unidos para países desenvolvidos é negativo, enquanto -para países em desenvolvimento - é positivo. Li, Ma e Xu (2015) analisaram a reação dos exportadores chineses às variações da taxa de câmbio relativas à moeda local. A partir de seu estudo, constataram ser pequena a resposta do preço das exportações em relação à variação cambial, enquanto a resposta ao volume é moderada. Asteriou, Masatci e Pilbeam (2016) investigaram a volatilidade da taxa de câmbio e sua relação com o volume do comércio internacional do México, da Indonésia, da Nigéria e da Turquia no longo e curto prazos. No longo, não encontraram vínculo entre a volatilidade da taxa de câmbio e as atividades comerciais internacionais, exceto na Turquia, onde tal relação, em todo o caso, mostrou-se fraca. No curto prazo, detectaram uma relação causal significativa entre a volatilidade e a demanda de importação/exportação, no caso da Indonésia e do México. Encontraram, para a Nigéria, causalidade unidirecional da demanda de exportação para a volatilidade, enquanto - para a Turquia - não detectaram causalidade alguma entre a volatilidade e a demanda de importação/exportação.

Bahmani-Oskooee e Harvey (2017), em estudo com foco em Cingapura, concluem que a volatilidade da taxa de câmbio tem efeitos significativos de curto 
prazo em 70 de 156 indústrias exportadoras e em 73 de 155 importadoras. No entanto, os efeitos de curto prazo duram no longo prazo apenas em 46 indústrias exportadoras e em 36 importadoras.

Tais resultados, bastante diversos, bem como a ausência de estudos com foco no Brasil apontam para a necessidade de mais pesquisas sobre a questão de forma mais aprofundada e rica. É precisamente nesse contexto que se encaixa este estudo.

\subsection{Efeitos da volatilidade da taxa de câmbio e o desenvolvimento dos países}

Segundo Takaenda, Tsheole e Aziakpono (2006), o impacto da volatilidade do câmbio no comércio exterior tem recebido mais atenção de pesquisas em países desenvolvidos do que naqueles em desenvolvimento, o que sugere a potencial relevância de tal diferenciação. A seguir, destacamos alguns autores que olharam o tema com foco no nível de desenvolvimento dos países analisados.

Chowdhury (1993) encontrou efeito negativo da volatilidade da taxa de câmbio no fluxo de comércio bilateral entre os países desenvolvidos pertencentes ao G-7, ao passo que Aristotelous (2001) não encontrou nenhum impacto das exportações britânicas para os Estados Unidos (ambos do G7). Por sua vez, Fountas e Bredin
(1997) encontraram efeitos diferentes nas exportações agregadas e setoriais da Irlanda para a União Europeia: nenhum impacto significativo no curto prazo, mas positivo e significativo no longo.

De Vitta e Abbot (2004) pesquisaram a volatilidade na exportação agregada dos Estados Unidos para um conjunto de países desenvolvidos, detectando um efeito negativo para o Reino Unido e a Alemanha, positivo para o Japão e nenhum efeito significativo para o Canadá. Byrne, Darby e MacDonald (2008), ao pesquisar a exportação tanto de produtos homogêneos quanto de diferenciados, de seis países desenvolvidos (Reino Unido, Alemanha, França, Itália, Holanda e Espanha), para os Estados Unidos, concluíram que os itens homogêneos não sofreram impacto e os diferenciados, efeitos negativos.

Há também estudos sobre países em desenvolvimento. Por exemplo, Altintas, Cetin e Oz (2011) encontraram efeito positivo da volatilidade nas exportações agregadas da Turquia. Bakhromov (2011), por sua vez, encontrou um impacto negativo nas exportações agregadas do Uzbequistão. Ekanayake, Thaver e Plante (2012) encontraram um efeito positivo das exportações da África do Sul para a União Europeia no longo prazo e negativo no curto. 
Wang e Barret (2007) estudaram as exportações setoriais do Taiwan para os Estados Unidos, encontrando efeito negativo na agricultura e nenhum efeito entre os outros setores analisados. Caglayan e Di (2010), por seu turno, voltaram-se para países desenvolvidos e em desenvolvimento: enquanto nestes o efeito foi negativo, naqueles foi positivo.

Em um estudo sobre o Brasil, Bittencourt e Campos (2014) verificaram se a instabilidade da taxa de câmbio afetou o fluxo de comércio de exportação e importação setoriais do Brasil com cinco de seus principais parceiros comerciais China, Argentina, Holanda, Japão e Chile , encontrando um resultado negativo para todos os setores pesquisados. Silva, Freitas e Mattos (2016) buscaram identificar o impacto da volatilidade da taxa de câmbio real bilateral sobre o fluxo de comércio entre países da América do Sul. Os resultados sugerem uma redução de atividades no comercio internacional por conta da exposição adicional ao risco cambial.

Silva e Bittencourt (2017) buscaram analisar o efeito da volatilidade da taxa de câmbio especificamente sobre o comércio intraindustrial entre os países do Mercosul. Os resultados da pesquisa apontam que tal comércio foi afetado positivamente durante o período analisado. De Vita e Abbott
(2004), reforçados por Baum e Caglayan (2010), concordam que os resultados da literatura, mesmo se considerado o nível de desenvolvimento dos países estudados, são mesclados, concluindo, portanto, que mais investigações se fazem necessárias.

\section{METODOLOGIA}

Foram coletados, nas bases IPEADATA, mantidas pelo Instituto de Pesquisa Econômica Aplicada (IPEA), dados da exportação brasileira por meio do índice de exportações (base $2006=100$, indicador do volume exportado), organizados por produtos e setores. Além do caráter inovador dessa segmentação em termos de pesquisa, ela gera maior poder informacional, contemplando vários produtos e setores relevantes da economia brasileira. Os setores e produtos pesquisados foram um total de sete: combustíveis; produtos manufaturados; bebidas; celulose, papel e produtos de papel; máquinas e equipamentos; produtos de metalurgia; e produtos alimentícios em geral. O período de abrangência dos dados vai do primeiro trimestre de 1999 ao terceiro trimestre de 2016. O ano de 1999 foi escolhido porque foi exatamente quando o Brasil passou a usar a política de câmbio flutuante. Anteriormente, a política de câmbio variou entre câmbio fixo e banda cambial. Entendemos que misturar períodos 
com políticas cambiais tão distintas não seria benéfico para a análise.

Com base no volume mensal das exportações, calculou-se a média trimestral e então tomou-se o logaritmo natural de tais médias. O uso de logaritmos é um procedimento extremamente usual na literatura (TAKAENDA; TSHEOLE; AZIAKPONO, 2006, e SERENIS; TSOUNIS, 2014 para citar apenas dois exemplos) e corrige distorções quando se lida com dados de grande escala e diferentes ordens de grandeza (como é o caso de nossas variáveis dependentes). Além disso, o uso de logaritmos normalmente diminui problemas de heteroscedasticidade e de assimetria, bem como lineariza relações entre variáveis medidas em escalas de naturezas distintas. Tais efeitos são importantes quando se utilizam modelos baseados em regressões lineares (BUENO, 2012).

Para as variáveis independentes adotadas, foram usadas a taxa de câmbio real obtida no website do IPEADATA e a de câmbio nominal obtida na base de dados do Banco Central. A taxa de câmbio efetiva real mensal usada no estudo foi a série denominada INPC - exportações - índice (base $2010=100)$.

Quanto à taxa de câmbio nominal, esta diz respeito ao câmbio diário da moeda brasileira. Como se considerou um período trimestral para os cálculos, foi feita a estimativa com 63 observações, referentes aos dias úteis do trimestre. A partir desses dados, foram calculadas quatro medidas de volatilidade: duas da taxa de câmbio real (VCR1 e VCR2) e duas da de câmbio nominal (VCN1 e VCN2), conforme especificações abaixo:

(I) VCR1: desvio-padrão da taxa de câmbio real de 2 trimestres;

(II) VCR2: desvio-padrão da taxa de câmbio real de 4 trimestres;

(III) VCN1: desvio-padrão da taxa de câmbio nominal de 2 trimestres;

(IV) VCN2: desvio-padrão da taxa de câmbio nominal de 4 trimestres.

Utilizar o desvio-padrão como proxy para a volatilidade de um ativo financeiro é normalmente a primeira escolha na literatura de finanças nacional e internacional, e o embasamento teórico para tal pode ser encontrado em Hull (2015). Para o cálculo das variáveis independentes VCN1 e VCR1, há um total de 69 observações, enquanto, para as variáveis VCN2 e VCR2, têm-se 67 observações. O uso das quatro métricas distintas de volatilidade visa capturar o efeito das variações da taxa de câmbio, real e nominal, que possam influenciar os produtos ou os setores pesquisados das exportações 
brasileiras, além de prover maior robustez à pesquisa.

Adotou-se como variável de controle nas estimativas o logaritmo dos termos de trocas trimestrais (TT), calculado a partir dos dados mensais coletados junto ao IPEADATA de forma análoga com o que foi feito com o volume de exportações. A escolha dessa variável de controle seguiu Bakhromov (2011). Outra variável utilizada como controle foi o PIB trimestral do Brasil a preço de 1995 (base $1995=100$ ), coletado nas bases de dados do IBGE. A partir dos dados trimestrais do PIB em valores absolutos, foi encontrado o logaritmo natural do PIB trimestral brasileiro.

Além dessas variáveis de controle, utilizaram-se três variáveis binárias (dummies) de dessazonalização a fim de modelar a sazonalidade dos trimestres dentro do próprio modelo, sendo tais variáveis referentes ao primeiro, ao segundo e ao terceiro trimestres. $\mathrm{O}$ quarto foi naturalmente controlado pelo termo constante (intercepto). As variáveis dependentes e independentes utilizadas no estudo são sumarizadas no Quadro 1.

\begin{tabular}{|l|l|}
\hline Quadro1 - Variáveis & \\
\hline \multirow{5}{*}{ Dependentes } & Exportações de celulose, papel e produtos de papel (ExpCelu) \\
& Exportações de máquinas e equipamentos (ExpMaqui) \\
Exportações de metalurgia (ExpMetal) & Exportações de produtos alimentícios (ExpAlim) \\
Exportações de produtos manufaturados (ExpManuf) & Exportações de combustíveis (ExpComb) \\
& Exportações de bebidas (ExpBeb) \\
\hline Independentes & Volatilidade cambial real 1 (VCR1) \\
& Volatilidade cambial real 2 (VCR2) \\
& Volatilidade cambial nominal 1 (VCN1) \\
& Volatilidade cambial nominal 2 (VCN2) \\
& Termos de troca trimestral (TT) \\
& PIB trimestral (PIB) \\
& $\begin{array}{l}\text { Dummy para dessazonalização temporal (D1) } \\
\text { Dummy para dessazonalização temporal (D2) } \\
\text { Dummy para dessazonalização temporal (D3) }\end{array}$ \\
\hline
\end{tabular}

Fonte: elaborado pelos autores.

A regressão dos dados foi executada pelo Método Generalizado dos Momentos (MGM) com o suporte do software EViews. Optou-se por uma estimativa por MGM para que problemas de endogenia (oriundos, por exemplo, de causalidades reversas e variáveis omitidas) pudessem ser tratados com variáveis instrumentais. Além disso, tal metodologia lida de forma mais eficaz com o problema da 
heteroscedasticidade. Destaca-se também que o estimador MGM apresenta melhores propriedades para pequenas amostras (BUENO, 2012), o que vem a ser o caso deste estudo.

Antes de estimar a regressão, as variáveis VCR1, VCR2, VCN1, VCN2, TT, PIB e as dependentes (relativas à exportação de produtos ou setores) devem ser analisadas para assegurar que nenhuma delas seja não estacionária, pois variáveis não estacionárias podem gerar uma regressão espúria. Desse modo, verificou-se a estacionariedade das séries por meio do teste de Dickey-Fuller Aumentado (ADF) (GUJARATI; PORTER, 2011). O teste ADF é o teste padrão para checar estacionariedade de séries temporais, ao passo que testes alternativos carecem do mesmo nível de reconhecimento na literatura. Por exemplo, o teste ADF é utilizado por Aristotelous (2001) e por Altintas, Cetin e Oz (2011), para mencionar apenas dois dos trabalhos citados por nós neste estudo.

Tabela 1 - Resultados do teste de raiz unitária

\begin{tabular}{|c|c|c|c|c|}
\hline & \multicolumn{2}{|c|}{ NÍVEL } & \multicolumn{2}{|c|}{$1^{\mathrm{a}}$ DIFERENÇA } \\
\hline SÉRIES E VARIÁVEIS & INTERCEPTO & $\begin{array}{l}\text { TENDÊNCIA E } \\
\text { INTERCEPTO }\end{array}$ & INTERCEPTO & $\begin{array}{l}\text { TENDÊNCIA E } \\
\text { INTERCEPTO }\end{array}$ \\
\hline \multicolumn{5}{|l|}{ COMBUSTÍVEIS } \\
\hline ln ExpComb & $-3,27(3) * * *$ & $-3,44(10)^{*}$ & $-11,65(0) * * *$ & $-11,63(0) * * *$ \\
\hline $\ln \mathrm{TT}$ & $-1,37(10)$ & $-1,40(10)$ & $-6,46(0)^{* * *}$ & $-6,49(0) * * *$ \\
\hline $\ln$ PIB & $-1,37(11)$ & $0,18(10)$ & $-3,15(10)^{* *}$ & $-3,47(11)^{*}$ \\
\hline VCR1 & $-6,44(0) * * *$ & $-6,78(0) * * *$ & $-9,82(1) * * *$ & $-9,75(1)^{* * *}$ \\
\hline VCR2 & $-3,00(4)^{* *}$ & $-3,60(4)^{* *}$ & $-6,82(2) * * *$ & $-6,76(2)^{* * *}$ \\
\hline VCN1 & $-3,66(4) * * *$ & $-3,63(4) * *$ & $-15,17(0) * * *$ & $-15,05(0)^{* * *}$ \\
\hline $\mathrm{VCN} 2$ & $-4,06(10) * * *$ & $-4,04(10)^{* * *}$ & $-6,46(10) * * *$ & $-6,38(10)^{* * * *}$ \\
\hline \multicolumn{5}{|l|}{ MANUFATURADOS } \\
\hline ln ExpManuf & $-1,98(10)$ & $-1,77(10)$ & $-3,24(2) * * *$ & $-9,31(2) * * *$ \\
\hline VCR1 & $-3,18(1)^{* *}$ & $-3,28(1)^{*}$ & $-17,33(0) * * *$ & $-17,22(0)^{* * *}$ \\
\hline VCR2 & $-3,00(2)^{* *}$ & $-3,32(2)^{*}$ & $-6,83(2)^{* * *}$ & $-6,76(2)^{* * *}$ \\
\hline VCN1 & $-3,66(1)^{* * *}$ & $-3,63(1) * *$ & $-15,17(0) * * *$ & $-15,05(0)^{* * *}$ \\
\hline VCN2 & $-4,06(4) * * *$ & $-4,04(4) * *$ & $-6,46(4) * * *$ & $-6,38(4)^{* * *}$ \\
\hline \multicolumn{5}{|l|}{ SETOR BEBIDAS } \\
\hline ln ExpBeb & $-5,30(2) * * *$ & $-5,19(2) * * *$ & $-9,67(2) * * *$ & $-9,68(2)^{* * *}$ \\
\hline VCR1 & $-3,13(4) * *$ & $-3,41(4)^{*}$ & $-9,82(2) * * *$ & $-9,75(2) * * *$ \\
\hline VCR2 & $-3,00(3)^{* *}$ & $-3,32(3) * * *$ & $-6,82(3) * * *$ & $-6,76(3) * * *$ \\
\hline VCN1 & $-3,66(10) * * *$ & $-3,63(10)^{* *}$ & $-9,91(10) * * *$ & $-9,84(10)^{* * *}$ \\
\hline $\mathrm{VCN} 2$ & $-4,06(2) * * *$ & $-4,04(2)^{* *}$ & $-6,46(2)^{* * *}$ & $-6,38(2)^{* * *}$ \\
\hline
\end{tabular}

(CONTINUA) 


\begin{tabular}{|c|c|c|c|c|}
\hline \multicolumn{5}{|c|}{ SETOR CELULOSE } \\
\hline In ExpCelu & $-0,61(10)$ & $-2,67(10)$ & $-13,29(10)^{* * *}$ & $-13,19(10)^{* * *}$ \\
\hline VCR1 & $-6,44(0) * *$ & $-6,78(0) * * *$ & $-17,33(0)^{* * *}$ & $-17,22(0) * * *$ \\
\hline VCR2 & $-3,00(2)^{* *}$ & $-3,32(2)^{*}$ & $-6,82(2) * * *$ & $-6,76(2)^{* * *}$ \\
\hline VCN1 & $-3,66(10)^{* *}$ & $-3,63(10)^{* *}$ & $-9,91(10)^{* * *}$ & $-9,18(10)^{* * *}$ \\
\hline $\mathrm{VCN} 2$ & $-4,06(4) * * *$ & $-4,04(4) * *$ & $-6,46(4) * * *$ & $-6,38(4) * * *$ \\
\hline \multicolumn{5}{|c|}{ SETOR MAQUINAS } \\
\hline In ExpMaqui & $-2,36(4)$ & $-2,28(4)$ & $-3,47(4) * * *$ & $-3,63(4)^{* *}$ \\
\hline VCR1 & $-3,13(4) * *$ & $-3,41(4)^{*}$ & $-9,82(4) * * *$ & $-9,75(4)^{* * *}$ \\
\hline $\mathrm{VCR} 2$ & $-3,00(4) * *$ & $-3,60(4)^{* *}$ & $-6,82(4) * * *$ & $-6,76(4) * * *$ \\
\hline VCN1 & $-3,66(4) * * *$ & $-3,63(4) * *$ & $-9,91(4) * * *$ & $-9,84(4) * * *$ \\
\hline $\mathrm{VCN} 2$ & $-4,06(4) * * *$ & $-4,04(4) * *$ & $-6,46(4) * * *$ & $-6,38(4)^{* * *}$ \\
\hline \multicolumn{5}{|c|}{ SETOR METALURGIA } \\
\hline ln ExpMetal & $-2,92(2) * *$ & $-3,62(2) * *$ & $-8,84(2) * * *$ & $-8,77(2) * * *$ \\
\hline VCR1 & $-3,18(2)^{* *}$ & $-3,28(2)^{*}$ & $-9,82(2) * * *$ & $-9,75(2) * * *$ \\
\hline VCR2 & $-3,00(2)^{* *}$ & $-3,32(2)^{*}$ & $-6,82(2) * * *$ & $-6,76(2) * * *$ \\
\hline VCN1 & $-3,66(2) * * *$ & $-3,63(2)^{* *}$ & $-9,91(2) * * *$ & $-9,84(2) * * *$ \\
\hline $\mathrm{VCN} 2$ & $-4,06(2) * * *$ & $-4,04(2)^{* *}$ & $-6,46(2)^{* * *}$ & $-6,38(2)^{* * *}$ \\
\hline \multicolumn{5}{|c|}{ SETOR ALIMENTÍCIOS } \\
\hline In ExpAlim & $-2,98(10)^{* *}$ & $-1,81(10)$ & $-4,81(10) * * *$ & $-11,52(10)^{* * *}$ \\
\hline VCR1 & $-3,18(10)^{* *}$ & $-3,41(10)^{*}$ & $-9,82(10)^{* * *}$ & $-9,75(10)^{* * *}$ \\
\hline VCR2 & $-2,47(10)$ & $-3,60(10)^{* *}$ & $-6,82(10) * * *$ & $-4,64(10) * * *$ \\
\hline VCN1 & $-3,66(10) * * *$ & $-3,63(10) * *$ & $-9,91(10) * * *$ & $-9,84(10)^{* * *}$ \\
\hline $\mathrm{VCN} 2$ & $-4,06(10) * * *$ & $-4,04(10) * *$ & $-6,46(10) * * *$ & $-6,38(10) * * *$ \\
\hline
\end{tabular}

Fonte: elaborado pelos autores.

Notas: ( ) representa o número de defasagens incluídas, que são selecionadas com base no Akaike Information Criteria (AIC) para teste ADF. O asterisco $(*)$ denota a significância estatística: *** 1\%, ** 5\%, *10\%. Os valores são fornecidos pelo Eviews.

Note-se que a rejeição na Tabela 1 (isto é, a presença de asteriscos) indica evidência de estacionariedade. Portanto, podemos concluir que a maioria das séries apresenta evidências de estacionariedade. Para aquelas sem tais evidências, trabalhamos com as suas primeiras diferenças (tradicional indicação na literatura), que apontaram, em todos os casos, estacionariedade. Dessa maneira, fomos capazes de seguir adiante em nossa análise.

\section{ANÁLISE DOS RESULTADOS}

As estimativas efetuadas pelo MGM neste estudo têm a intenção de verificar os efeitos que a volatilidade da taxa de câmbio pode causar nas exportações brasileiras. A Tabela 2 apresenta as estatísticas descritivas das variáveis adotadas neste estudo. 
Tabela 2 - Estatística Descritiva

\begin{tabular}{|c|c|c|c|c|c|c|}
\hline VARIÁVEIS & MÉDIA & MEDIANA & MÁXIMO & MÍNIMO & DESVIO & OBSERVAÇÕES \\
\hline$\underline{\ln \text { ExpComb } 1}$ & 4,44 & 4,61 & 5 & 3,15 & 0,47 & 69 \\
\hline In ExpComb 2 & 4,47 & 4,61 & 5 & 3,15 & 0,44 & 67 \\
\hline$\underline{\ln \text { ExpManuf } 1}$ & 4,34 & 4,38 & 4,68 & 3,82 & 0,23 & 69 \\
\hline ln ExpManuf 2 & 4,36 & 4,39 & 4,68 & 3,86 & 0,21 & 67 \\
\hline In ExpBeb 1 & 4,45 & 4,47 & 4,8 & 3,68 & 0,21 & 69 \\
\hline$\underline{\ln \text { ExpBeb } 2}$ & 4,47 & 4,48 & 4,8 & 3,94 & 0,18 & 67 \\
\hline$\underline{\text { ln ExpCelu } 1}$ & 4,6 & 4,65 & 5,19 & 3,77 & 0,36 & 69 \\
\hline$\underline{\ln \text { ExpCelu } 2}$ & 4,62 & 4,66 & 5,19 & 3,77 & 0,35 & 67 \\
\hline$\underline{\ln \text { ExpMaqui } 1}$ & 4,27 & 4,35 & 4,71 & 3,48 & 0,35 & 69 \\
\hline In ExpMaqui 2 & 4,29 & 4,36 & 4,71 & 3,59 & 0,33 & 67 \\
\hline$\underline{\ln \text { ExpMetal } 1}$ & 4,45 & 4,47 & 4,77 & 4,12 & 0,15 & 69 \\
\hline In ExpMetal 2 & 4,46 & 4,48 & 4,77 & 4,12 & 0,14 & 67 \\
\hline$\underline{\ln \text { ExpAlim } 1}$ & 4,5 & 4,58 & 4,89 & 3,63 & 0,3 & 69 \\
\hline$\underline{\ln \text { ExpAlim } 2}$ & 4,52 & 4,59 & 4,89 & 3,63 & 0,29 & 67 \\
\hline$\underline{\ln \text { PIB }}$ & 12,42 & 12,43 & 12,65 & 12,13 & 0,16 & 69 \\
\hline $\ln \mathrm{TT}$ & 4,64 & 4,62 & 4,87 & 4,51 & 0,1 & 69 \\
\hline VCR1 & 5,29 & 3,97 & 26,46 & 0,007 & 5,56 & 69 \\
\hline VCR2 & 8,45 & 5,84 & 31,92 & 1,04 & 6,51 & 67 \\
\hline VCN1 & 0,09 & 0,06 & 0,44 & 0 & 0,1 & 69 \\
\hline VCN2 & 0,16 & 0,12 & 0,6 & 0,01 & 0,12 & 67 \\
\hline
\end{tabular}

Fonte: elaboração própria.

Nota-se, na Tabela 2, que o número de observações se diferencia entre as variáveis dependentes (exportações de produtos e setores) e as independentes relativas a volatilidade, visto que, em alguns casos, os cálculos partem do desvio-padrão de 2 trimestres e, em outros, do desviopadrão de 4 trimestres.

Para estimar os efeitos da volatilidade da taxa de câmbio real (VCR1 e VCR2) e da volatilidade da taxa de câmbio nominal (VCN1 e VCN2) sobre as exportações brasileiras, foram apresentados, nas Tabelas 3 a 9, os resultados em diferentes produtos (combustíveis, manufaturados e bebidas) ou setores de exportação da economia brasileira (celulose, papel e produtos de papel, metalurgia, máquinas e equipamentos e produtos alimentícios).

A Tabela 3 apresenta os resultados das estimativas, mensurando os efeitos da volatilidade da taxa de câmbio (manifesta nas variáveis independentes) sobre a variável dependente ExpComb. Os resultados obtidos, para a variável de interesse volatilidade, não se mostraram estatisticamente significativos em nenhuma 
das regressões estimadas, daí a volatilidade

da taxa de câmbio não influenciar as

exportações brasileiras de combustíveis.

Tabela 3 - Regressão exportação de combustíveis

\begin{tabular}{|c|c|c|c|c|}
\hline \multicolumn{5}{|c|}{$\begin{array}{l}\text { Método generalizado dos momentos - MGM } \\
\text { Amostra: 1999T1 - 2016T3 } \\
\text { Matriz de ponderação dos estimadores: HAC (Bartlet Kernel, Newest-West janela fixa = 4000) } \\
\text { Erro padrão e covariância: matriz de ponderação HAC (Bartlet Kernel, Newest-West janela fixa = 4000) } \\
\text { Variável dependente: ExpComb }\end{array}$} \\
\hline Regressão & 1 & 2 & 3 & 4 \\
\hline VCR1 & $0,00(0,73)$ & & & \\
\hline VCR2 & & $0,00(0,74)$ & & \\
\hline VCN1 & & & $0,23(0,48)$ & \\
\hline VCN2 & & & & $0,22(0,55)$ \\
\hline $\ln \mathbf{T T}$ & $-0,54(0,36)$ & $-0,42(0,48)$ & $-0,44(0,46)$ & $-0,18(0,79)$ \\
\hline In PIB & $2,18 * * *(0,00)$ & $2,05 * * *(0,00)$ & $2,09 * * *(0,00)$ & $1,83 * * *(0,00)$ \\
\hline Dummy 1 & $-0,01(0,43)$ & $-0,00(0,99)$ & $-0,03(0,55)$ & $-0,00(0,94)$ \\
\hline Dummy 2 & $-0,04(0,78)$ & $-0,01(0,78)$ & $-0,01(0,78)$ & $-0,01(0,78)$ \\
\hline Dummy 3 & $0,03(0,50)$ & $-0,06(0,24)$ & $-0,04(0,38)$ & $0,06(0,23)$ \\
\hline Intercepto & $-20,11 * * *(0,00)$ & $-19,05^{* * *}(0,00)$ & $-19,50 * * *(0,00)$ & $-17,39 * * *(0,00)$ \\
\hline R2 Ajustado & 0,56 & 0,54 & 0,56 & 0,54 \\
\hline Ranking Inst. & 8 & 8 & 8 & 8 \\
\hline Estatística J & 9,05 & 8,52 & 8,99 & 8,46 \\
\hline Valor-p Est. J & 0,000 & 0,000 & 0,000 & 0,000 \\
\hline No Obs. & 69 & 67 & 69 & 67 \\
\hline
\end{tabular}

Fonte: elaboração própria.

Nota: $* * *$ significativo a $1 \%$, **significativo a $5 \%$ e *significativo a $10 \%$. Entre parênteses, valor p.

Os termos de troca e o PIB apresentaram efeitos significativos em muitas estimativas. Entretanto, por serem somente variáveis de controle e não objeto deste estudo, optou-se por não colocar as interpretações em relação aos resultados obtidos nas regressões estimadas.
Com a intenção de verificar se os efeitos da volatilidade da taxa de câmbio seguem a mesma direção da exportação de combustíveis, estimam-se os efeitos da volatilidade da taxa de câmbio na variável dependente ExpManuf, conforme Tabela 4. 
Tabela 4 - Regressão exportação de manufaturados

\begin{tabular}{|c|c|c|c|c|}
\hline \multicolumn{5}{|c|}{$\begin{array}{l}\text { Método generalizado dos momentos - GMM } \\
\text { Amostra: 1999T1 - 2016T3 } \\
\text { Matriz de ponderação dos estimadores: HAC (Bartlet Kernel, Newest-West janela fixa = 4000) } \\
\text { Erro padrão e covariância: matriz de ponderação HAC (Bartlet Kernel, Newest-West janela fixa = 4000) } \\
\text { Variável dependente: ExpManuf }\end{array}$} \\
\hline Regressão & 1 & 2 & 3 & 4 \\
\hline VCR1 & $-0,00 *(0,10)$ & & & \\
\hline VCR2 & & $-0,01 * * *(0,01)$ & & \\
\hline VCN1 & & & $-0,39(0,13)$ & \\
\hline VCN2 & & & & $-0,67 * *(0,02)$ \\
\hline $\ln \mathrm{TT}$ & $-0,60(0,27)$ & $-0,81(0,13)$ & $-0,68(0,22)$ & $-1,19 * *(0,05)$ \\
\hline In PIB & $0,98 * *(0,02)$ & $0,76 *(0,08)$ & $1,07 * * *(0,00)$ & $1,18 * * *(0,00)$ \\
\hline Dummy 1 & $-0,15 * * *(0,00)$ & $-0,19 * * *(0,00)$ & $-0,17 * * *(0,00)$ & $-0,17 * * *(0,00)$ \\
\hline Dummy 2 & $-0,07 * * *(0,02)$ & $-0,10 * * *(0,00)$ & $-0,08 * *(0,01)$ & $-0,12 * * *(0,00)$ \\
\hline Dummy 3 & $-0,02(0,30)$ & $-0,03(0,20)$ & $-0,04 *(0,07)$ & $-0,05^{*}(0,07)$ \\
\hline Intercepto & $-4,94(0,17)$ & $-1,14(0,77)$ & $-5,73(0,07)$ & $-4,57(0,18)$ \\
\hline R2 Ajustado & 0,37 & 0,38 & 0,37 & 0,39 \\
\hline Ranking Inst. & 8 & 8 & 8 & 8 \\
\hline Estatística J & 11,09 & 10,53 & 11,53 & 11,43 \\
\hline Valor-p Est. J & 0,000 & 0,000 & 0,000 & 0,000 \\
\hline $\mathbf{N}^{0}$ Obs. & 69 & 67 & 69 & 67 \\
\hline
\end{tabular}

Fonte: elaboração própria.

Nota: $* * *$ significativo a $1 \%, * *$ significativo a $5 \%$ e $*$ significativo a $10 \%$. Entre parênteses, valor p.

Os resultados apresentados na Tabela 4 sugerem que a volatilidade cambial impacta negativamente as exportações de manufaturados do Brasil em três das quatro estimativas verificadas. A volatilidade da taxa de câmbio mostrou-se estatisticamente significativa ao nível de $10 \%$ na VCR1, enquanto o resultado na VCR2 foi mais interessante: significativo a $1 \%$. Quanto à VCN2, foi encontrado um nível de significância de 5\% da volatilidade da taxa de câmbio. Convém destacar que todos os coeficientes apresentaram resultados negativos, ou seja, um aumento da volatilidade da taxa de câmbio diminui as exportações de manufaturados.

Quanto aos efeitos da volatilidade da taxa de câmbio nominal e real nas exportações de bebidas (ver Tabela 5), os resultados mostraram-se estatisticamente significativos em todas as estimativas efetuadas: nível de significância de $1 \%$ na 
VCR1 e na VCN1, estatisticamente significativos ao nível de 5\% na VCR2 e de $10 \%$ na VCN2. Como todos os coeficientes apresentam resultados negativos, são semelhantes aos resultados negativos encontrados na Tabela 4.

Tabela 5 - Exportações de bebidas

\begin{tabular}{|c|c|c|c|c|}
\hline \multicolumn{5}{|c|}{$\begin{array}{l}\text { Método generalizado dos momentos - GMM } \\
\text { Amostra: 1999T1 - 2016T3 } \\
\text { Matriz de ponderação dos estimadores: HAC (Bartlet Kernel, Newest-West janela fixa = 4000) } \\
\text { Erro padrão e covariância: matriz de ponderação HAC (Bartlet Kernel, Newest-West janela fixa = 4000) } \\
\text { Variável dependente: ExpBeb }\end{array}$} \\
\hline Regressão & 1 & 2 & 3 & 4 \\
\hline VCR1 & $-0,01 * * *(0,00)$ & & & \\
\hline VCR2 & & $-0,01 * *(0,05)$ & & \\
\hline VCN1 & & & $-0,77 * * *(0,00)$ & \\
\hline VCN2 & & & & $-0,42 *(0,09)$ \\
\hline $\ln \mathrm{TT}$ & $-1,19 * * *(0,00)$ & $-1,29 * * *(0,00)$ & $-1,44 * * *(0,00)$ & $-1,35 * * *(0,00)$ \\
\hline $\ln$ PIB & $0,68 *(0,05)$ & $0,51 *(0,06)$ & $0,98 * *(0,01)$ & $0,74 * *(0,04)$ \\
\hline Dummy 1 & $-0,20 * * *(0,00)$ & $-0,16 * * *(0,00)$ & $-0,23 * * *(0,00)$ & $-0,16 * * *(0,00)$ \\
\hline Dummy 2 & $-0,11 * * *(0,03)$ & $-0,10 * *(0,02)$ & $-0,13 * *(0,01)$ & $-0,12 * *(0,02)$ \\
\hline Dummy 3 & $-0,04(0,28)$ & $-0,02(0,59)$ & $-0,07 *(0,08)$ & $-0,03(0,43)$ \\
\hline Intercepto & $1,66 * *(0,01)$ & $4,31(0,10)$ & $-0,90(0,78)$ & $1,73(0,54)$ \\
\hline R2 Ajustado & 0,27 & 0,27 & 0,26 & 0,21 \\
\hline Ranking Inst. & 8 & 8 & 8 & 8 \\
\hline Estatística J & 7,27 & 7,50 & 6,98 & 7,00 \\
\hline Valor-p Est. J & 0,000 & 0,000 & 0,000 & 0,000 \\
\hline $\mathbf{N}^{0}$ Obs. & 69 & 67 & 69 & 67 \\
\hline
\end{tabular}

Fonte: elaboração própria.

Nota: $* * *$ significativo a $1 \%, * *$ significativo a $5 \%$ e $*$ significativo a $10 \%$. Entre parênteses, valor p.

A fim de aumentar as respostas e a celulose, máquinas e equipamentos, robustez dos resultados dos efeitos da metalurgia e produtos alimentícios. O volatilidade da taxa de câmbio real e primeiro setor a ser estimado encontra-se nominal nas exportações brasileiras, especificado na Tabela 6. estimaram-se tais efeitos nos setores de 
Tabela 6 - Exportações setor celulose, papel e produtos de papel.

\begin{tabular}{|c|c|c|c|c|}
\hline \multicolumn{5}{|c|}{$\begin{array}{l}\text { Método generalizado dos momentos - GMM } \\
\text { Amostra: 1999T1 - 2016T3 } \\
\text { Matriz de ponderação dos estimadores: HAC (Bartlet Kernel, Newest-West janela fixa = 4000) } \\
\text { Erro padrão e covariância: matriz de ponderação HAC (Bartlet Kernel, Newest-West janela fixa = 4000) } \\
\text { Variável dependente: ExpCelu }\end{array}$} \\
\hline Regressão & 1 & 2 & 3 & 4 \\
\hline VCR1 & $-0,00(0,63)$ & & & \\
\hline VCR2 & & $0,00(0,93)$ & & \\
\hline VCN1 & & & $-0,05(0,59)$ & \\
\hline VCN2 & & & & $0,07(0,53)$ \\
\hline $\ln T T$ & $-1,26 * * *(0,00)$ & $-1,25 * * *(0,00)$ & $-1,28 * * *(0,00)$ & $-1,15 * * *(0,00)$ \\
\hline In PIB & $2,60 * * *(0,00)$ & $2,62 * * *(0,00)$ & $2,62 * * *(0,00)$ & $2,57 * * *(0,00)$ \\
\hline Dummy 1 & $0,07 * *(0,01)$ & $0,07 * *(0,02)$ & $0,06 * *(0,02)$ & $0,07 * *(0,01)$ \\
\hline Dummy 2 & $0,02(0,43)$ & $0,02(0,48)$ & $0,02(0,49)$ & $0,02(0,43)$ \\
\hline Dummy 3 & $-0,03(0,11)$ & $-0,03(0,20)$ & $-0,04(0,11)$ & $-0,02(0,30)$ \\
\hline Intercepto & $-21,84 * * *(0,00)$ & $-22,16 * * *(0,00)$ & $-21,97 * * *(0,00)$ & $-22,00 * * *(0,00)$ \\
\hline R2 Ajustado & 0,91 & 0,91 & 0,91 & 0,91 \\
\hline Ranking Inst. & 8 & 8 & 8 & 8 \\
\hline Estatística J & 10,66 & 10,75 & 10,66 & 10,81 \\
\hline Valor-p Est. J & 0,000 & 0,000 & 0,000 & 0,000 \\
\hline $\mathbf{N}^{\circ}$ Obs. & 69 & 67 & 69 & 67 \\
\hline
\end{tabular}

Fonte: elaboração própria.

Nota: $* * *$ significativo a $1 \%, * *$ significativo a $5 \%$ e $*$ significativo a $10 \%$. Entre parênteses, valor p.

Os efeitos da volatilidade da taxa de câmbio real e nominal não apresentaram nenhum efeito nas exportações do setor celulose, papel e produtos de papel, mesmo considerando uma significância de $10 \%$. Por ser um setor bastante exportador no Brasil, pode ser que as empresas não tenham a opção em vender ou não para o mercado externo, visto que muitos contratos de compra no setor são fechados antecipadamente. Portanto, a volatilidade não é fator crucial para a quantidade de celulose e derivados exportados.

Analisando a Tabela 7, verificam-se os efeitos negativos da volatilidade da taxa de câmbio nas exportações do setor máquinas e equipamentos. A volatilidade da taxa de câmbio real apresenta efeito significativo ao nível de 5\% na VCR1 e de $1 \%$ na VCR2, VCN1 e VCN2, alterando somente os valores dos coeficientes. Entretanto, os sinais dos coeficientes são 
negativos, indicando que um aumento na volatilidade da taxa de câmbio diminui as exportações para o setor de máquinas e equipamentos.

Tabela 7 - Exportações setor máquinas e equipamentos

\begin{tabular}{|c|c|c|c|c|}
\hline \multicolumn{5}{|c|}{$\begin{array}{l}\text { Método generalizado dos momentos - GMM } \\
\text { Amostra: } 1999 \mathrm{~T} 1 \text { - 2016T3 } \\
\text { Matriz de ponderação dos estimadores: HAC (Bartlet Kernel, Newest-West janela fixa = 4000) } \\
\text { Erro padrão e covariância: matriz de ponderação HAC (Bartlet Kernel, Newest-West janela fixa = 4000) } \\
\text { Variável dependente: ExpMaqui }\end{array}$} \\
\hline Regressão & 1 & 2 & 3 & 4 \\
\hline VCR1 & $-0,01 * *(0,05)$ & & & \\
\hline VCR2 & & $-0,02 * * *(0,01)$ & & \\
\hline VCN1 & & & $-0,71 * * *(0,00)$ & \\
\hline VCN2 & & & & $-1,06 * * *(0,01)$ \\
\hline $\ln \mathrm{TT}$ & $-0,19(0,82)$ & $-0,51(0,53)$ & $-0,34(0,68)$ & $-1,05(0,26)$ \\
\hline In PIB & $1,14 *(0,08)$ & $0,66(0,32)$ & $1,34 * *(0,03)$ & $1,34 * *(0,04)$ \\
\hline Dummy 1 & $-0,12 * *(0,02)$ & $-0,17 * * *(0,00)$ & $-0,15 * * *(0,00)$ & $-0,15 * * *(0,00)$ \\
\hline Dummy 2 & $-0,06(0,20)$ & $-0,12 * *(0,01)$ & $-0,07(0,12)$ & $-0,15 * * *(0,00)$ \\
\hline Dummy 3 & $-0,05(0,17)$ & $-0,07(0,10)$ & $-0,08 * *(0,02)$ & $-0,10 * *(0,03)$ \\
\hline Intercepto & $-8,89(0,11)$ & $-1,32(0,83)$ & $-10,70 * *(0,03)$ & $-7,18(0,18)$ \\
\hline R2 Ajustado & 0,34 & 0,29 & 0,34 & 0,32 \\
\hline Ranking Inst. & 8 & 8 & 8 & 8 \\
\hline Estatística J & 12,14 & 11,03 & 12,64 & 12,65 \\
\hline Valor-p Est. J & 0,000 & 0,000 & 0,000 & 0,000 \\
\hline $\mathbf{N}^{\mathbf{0}}$ Obs. & 69 & 67 & 69 & 67 \\
\hline
\end{tabular}

Fonte: elaboração própria.

Nota: $* * *$ significativo a $1 \%, * *$ significativo a $5 \%$ e *significativo a $10 \%$. Entre parênteses, valor p.

Os efeitos da volatilidade da taxa de câmbio nas exportações do setor de metalurgia no Brasil não são tão claros (ver Tabela 8), tendo em vista um efeito negativo da volatilidade da taxa de câmbio nominal (VCN1) nas exportações de tal setor, sendo o nível de significância de 10\%, ao passo que a volatilidade VCR1, a VCR2 e a VCN2 não apresentam nenhum efeito. 
Tabela 8 - Exportações setor metalurgia

\begin{tabular}{|c|c|c|c|c|}
\hline \multicolumn{5}{|c|}{$\begin{array}{l}\text { Método generalizado dos momentos - GMM } \\
\text { Amostra: 1999T1 - } 2016 \mathrm{~T} 3 \\
\text { Matriz de ponderação dos estimadores: HAC (Bartlet Kernel, Newest-West janela fixa = 4000) } \\
\text { Erro padrão e covariância: matriz de ponderação HAC (Bartlet Kernel, Newest-West janela fixa = 4000) } \\
\text { Variável dependente: ExpMetal }\end{array}$} \\
\hline Regressão & 1 & 2 & 3 & 4 \\
\hline VCR1 & $0,00(0,95)$ & & & \\
\hline VCR2 & & $-0,00(0,75)$ & & \\
\hline VCN1 & & & $-0,71 *(0,07)$ & \\
\hline VCN2 & & & & $-0,00(0,99)$ \\
\hline $\ln \mathbf{T T}$ & $-1,32 * * *(0,00)$ & $-1,36 * * *(0,00)$ & $-0,34(0,68)$ & $-1,34 * * *(0,00)$ \\
\hline In PIB & $1,17 * * *(0,00)$ & $1,11 * * *(0,00)$ & $1,34 * *(0,03)$ & $1,12 * * *(0,00)$ \\
\hline Dummy 1 & $0,02(0,27)$ & $0,02(0,37)$ & $-0,015 * * *(0,00)$ & $0,02(0,30)$ \\
\hline Dummy 2 & $-0,05 * *(0,03)$ & $-0,05 * *(0,04)$ & $-0,07(0,12)$ & $-0,05 *(0,06)$ \\
\hline Dummy 3 & $-0,00(0,82)$ & $0,01(0,63)$ & $-0,08 * *(0,02)$ & $0,01(0,62)$ \\
\hline Intercepto & $-3,89 * *(0,01)$ & $-3,03(0,10)$ & $-10,70 * *(0,03)$ & $-3,26(0,05)$ \\
\hline R2 Ajustado & 0,41 & 0,39 & 0,34 & 0,38 \\
\hline Ranking Inst. & 8 & 8 & 8 & 8 \\
\hline Estatística J & 12,28 & 12,17 & 12,64 & 11,98 \\
\hline Valor-p Est. J & 0,000 & 0,000 & 0,000 & 0,000 \\
\hline $\mathbf{N}^{\mathbf{o}}$ Obs. & 69 & 67 & 69 & 67 \\
\hline
\end{tabular}

Fonte: elaboração própria.

Nota: $* * *$ significativo a $1 \%, * *$ significativo a $5 \%$ e $*$ significativo a $10 \%$. Entre parênteses, valor p.

Por fim, verificam-se, na Tabela 9, os efeitos da volatilidade da taxa de câmbio no setor produtos alimentícios.

Tabela 9 - Exportações setor produtos alimentícios

\begin{tabular}{|c|c|c|c|c|}
\hline \multicolumn{5}{|c|}{$\begin{array}{l}\text { Método generalizado dos momentos - GMM } \\
\text { Amostra: 1999T1 - 2016T3 } \\
\text { Matriz de ponderação dos estimadores: HAC (Bartlet Kernel, Newest-West janela fixa = 4000) } \\
\text { Erro padrão e covariância: matriz de ponderação HAC (Bartlet Kernel, Newest-West janela fixa = 4000) } \\
\text { Variável dependente: ExpAlim }\end{array}$} \\
\hline Regressão & 1 & 2 & 3 & 4 \\
\hline VCR1 & $-0,00 * * *(0,00)$ & & & \\
\hline VCR2 & & $-0,00(0,31)$ & & \\
\hline VCN1 & & & $-0,14(0,55)$ & \\
\hline
\end{tabular}




\begin{tabular}{|c|c|c|c|c|}
\hline VCN2 & & & & $-0,17(0,49)$ \\
\hline $\ln T T$ & $-0,95 * *(0,05)$ & $-0,98 * *(0,04)$ & $-0,95 * *(0,04)$ & $-1,02 * *(0,04)$ \\
\hline In PIB & $1,65 * * *(0,00)$ & $1,49 * * *(0,00)$ & $1,68 * * *(0,00)$ & $1,59 * * *(0,00)$ \\
\hline Dummy 1 & $-0,18 * * *(0,00)$ & $-0,19 * * *(0,00)$ & $-0,08 * * *(0,00)$ & $-0,18 * * *(0,00)$ \\
\hline Dummy 2 & $-0,08 * * *(0,00)$ & $-0,07 * *(0,01)$ & $-0,08 * * *(0,00)$ & $-0,08 * *(0,01)$ \\
\hline Dummy 3 & $0,05 * *(0,04)$ & $0,07 * * *(0,00)$ & $0,04(0,13)$ & $0,06 * *(0,03)$ \\
\hline Intercepto & $-11,57 * * *(0,00)$ & $-9,33 * *(0,01)$ & $-11,85 * *(0,00)$ & $-10,38 * * *(0,00)$ \\
\hline R2 Ajustado & 0,69 & 0,66 & 0,69 & 0,66 \\
\hline Ranking Inst. & 8 & 8 & 8 & 8 \\
\hline Estatística J & 9,21 & 7,96 & 9,23 & 7,97 \\
\hline Valor-p Est. J & 0,000 & 0,000 & 0,000 & 0,000 \\
\hline $\mathbf{N}^{\circ}$ Obs. & 69 & 67 & 69 & 67 \\
\hline
\end{tabular}

Fonte: elaboração própria.

Nota: $* * *$ significativo a $1 \%, * *$ significativo a $5 \%$ e *significativo a $10 \%$. Entre parênteses, valor p.

Os resultados das estimativas dos efeitos da volatilidade da taxa de câmbio real e nominal nas exportações de produtos alimentícios não apresentam nenhum efeito. Tais resultados são semelhantes àqueles encontrados nas estimativas da volatilidade no setor de metalurgia e no setor de celulose, papel e produtos de papel. Pode ser que o setor alimentício tenha a opção de vender no mercado interno ao verificar que a volatilidade da taxa de câmbio está afetando a sua lucratividade.

Como podemos perceber, os resultados aqui encontrados no mercado brasileiro dependem do setor da economia analisado: ora não houve evidências de impacto da volatilidade cambial nas exportações do setor, ora a evidência mostrou-se estatisticamente significativa. Essa conclusão, em que pese ser original para o mercado brasileiro, não é única na literatura mundial. Por exemplo, Byrne, Darby e MacDonald (2008), estudando o mercado estadunidense, encontraram diferentes resultados para diferentes indústrias. Por sua vez, Fountas e Bredin (1997) também já haviam encontrado tais resultados analisando as exportações irlandesas para a União Europeia. Em consequência, acreditamos que estudos que agreguem características setoriais para entendermos as reais razões de haver efeito ou não em determinado setor econômico podem contribuir para o presente debate de maneira relevante e original. 


\section{CONSIDERAÇÕES FINAIS}

Este estudo buscou identificar os efeitos da volatilidade da taxa de câmbio sobre as exportações brasileiras, em diversos de seus produtos e setores. A literatura acerca do tema sugere que a volatilidade cambial influencia as exportações de vários países, de modo negativo em alguns casos e nulo em outros. Alguns estudos foram inconclusivos, ou seja, encontraram efeito positivo em algumas situações e negativo em outras. Com a intenção de verificar os efeitos da volatilidade da taxa de câmbio nas exportações brasileiras, foram calculadas quatro proxies de volatilidade como variáveis independentes. Como variáveis dependentes, utilizaram-se dados de exportações de alguns setores e produtos da economia brasileira. Os resultados obtidos não foram uníssonos, tendo sido encontrados efeitos diferentes para diferentes produtos e setores exportadores, o que vai ao encontro da literatura internacional.

Os resultados encontrados no produto bebidas e no setor máquinas e equipamentos apresentaram efeitos negativos e estatisticamente significativos para todas as métricas utilizadas. Quanto aos produtos combustíveis, bem como ao setor celulose, papel e produtos de papel, além do setor alimentício, não foram encontrados indícios de efeitos com nenhuma das métricas utilizadas. Porém, com relação aos produtos manufaturados, os resultados se mostraram significativos (e negativos) em três das quatro métricas, enquanto - no setor de metalurgia - a significância estatística se deu com somente uma métrica (VCN3).

Diante dos resultados expostos, verificou-se que a volatilidade da taxa de câmbio pode afetar as exportações brasileiras de forma negativa em alguns casos. Consequentemente, patamares mais estáveis para as taxas de câmbio podem ajudar as exportações brasileiras, aumentando nossa competitividade no mercado mundial e ajudando a balança comercial brasileira.

Uma limitação deste estudo diz respeito à quantidade de dados disponíveis do câmbio, pois o Brasil se tornou um país com regime de câmbio flutuante somente em 1999, impossibilitando uma maior quantidade de dados para serem analisados. Como sugestão de pesquisa, entendemos ser relevante pesquisar os efeitos da volatilidade da taxa de câmbio também nas importações brasileiras, considerando produtos e setores da economia, bem como no próprio saldo da balança comercial. Pode-se também pesquisar a influência da volatilidade da taxa de câmbio no curto e no 
longo prazos, considerando técnicas de

cointegração e mecanismos de correção de erros. 


\section{REFERÊNCIAS}

ALTINTAŞ, H.; CETIN, R.; ÖZ, B. The Impact of Exchange Rate Volatility on Turkish Exports: 1993-2009. South East European Journal of Economics and Business, v. 6, n. 2, p. 71-81, 1 jan. 2011.

ARISTOTELOUS, K. Exchange-rate volatility, exchange-rate regime, and trade volume: Evidence from the UK-US export function (1889-1999). Economics Letters, v. 72, n. 1, p. 8794, 1 jul. 2001.

ARIZE, A. C.; OSANG, T.; AND SLOTTJE, D. J. Exchange volatility and foreign trade: Evidence from thirteen LDCs. Journal of Business and Economic Statistics, v. 18, n. 1, p. 10-17, jan. 2000.

Exchange-rate volatility in Latin America and its impact on foreign trade. International Review of Economics and Finance, v. 17, n. 1, p. 33-44, 1 jan. 2008.

ASTERIOU, D.; MASATCI, K.; PILBEAM, K. Exchange rate volatility and international trade: International evidence from the MINT countries. Economic Modelling, v. 58, p. 133140, nov. 2016.

BAHMANI-OSKOOEE, M.; HARVEY, H. Exchange rate volatility and its impact on commodity trade flows between singapore and malaysia. Journal Of Economic Development, v. 17, n. 1, p. 17-33, 2017.

BAKHROMOV, N. The Exchange Rate Volatility and the Trade Balance: Case of Uzbekistan. Journal of Applied Economics and Business Research JAEBR, v. 1, n. 3, p. 149-161, 2011.

BAUM, C. F.; CAGLAYAN, M. On the sensitivity of the volume and volatility of bilateral trade flows to exchange rate uncertainty. Journal of International Money and Finance, v. 29, n. 1, p. 79-93, 1 fev. 2010.

BITTENCOURT, G. M.; CAMPOS, A. C. Efeitos da instabilidade da taxa de câmbio no comércio setorial entre Brasil e seus principais parceiros comerciais. Economia Aplicada, v. 18, n. 4, p. 657-678, dez. 2014.

BUENO, R. DE L. DA S. Econometria de Séries Temporais. 2. ed. 2008.

BYRNE, J. P.; DARBY, J.; MACDONALD, R. US trade and exchange rate volatility: A real 
sectoral bilateral analysis. Journal of Macroeconomics, v. 30, n. 1, p. 238-259, 1 mar. 2008.

CAGLAYAN, M.; DI, J. Does Real Exchange Rate Volatility Affect Sectoral Trade Flows? Southern Economic Journal, v. 77, p. 313-335, October 2010.

CHOWDHURY, A. Does Exchange Rate Variability Depress Trade Flows? Evidence From Error Correction Models. Review of Economics and Statistics, v. 75, n. 4, p. 700-706, 1993.

DE BIASI DA SILVA, C.; VAZ, M.; BITTENCOURT, L. O comércio intra-industrial do Mercosul e a volatilidade da taxa de câmbio. In: XX ENCONTRO DE ECONOMIA DA REGIÃO SUL, 2017, Porto Alegre. Disponível em: $<$ https://www.anpec.org.br/sul/2017/submissao/files_I/i5-

1f402e0fd994cb90ed8c44f97775f534.pdf>. Acesso em: 3 nov. 2017.

EKANAYAKE, E. M.; THAVER, R. L.; PLANTE, D. The Effects of Exchange Rate Volatility on South Africa's Trade with the European Union. The International Journal of Business and Finance Research, v. 6 (3), p. 13-26, 2012.

FOUNTAS, S.; BREDIN, D. Exchange Rate Volatility and Exports: The Case of Ireland. Department of Economics, National University of Ireland, Galway, v. 16, p. 301-304, May 1997.

GUJARATI, D. N.; PORTER, D. C. Econometria básica. 5. ed. Porto Alegre: AMGH, 2011.

HSU, K. C.; CHIANG, H. C. The threshold effects of exchange rate volatility on exports: Evidence from US bilateral exports. Journal of International Trade and Economic Development, v. 20, n. 1, p. 113-128, fev. 2011.

HULL, J. Options, Futures, and Other Derivatives. 9. ed. Pearson Education, 2015.

LI, H.; MA, H.; XU, Y. How do exchange rate movements affect Chinese exports? - A firmlevel investigation. Journal of International Economics, v. 97, n. 1, p. 148-161, 1 set. 2015.

OZTURK, I.; KALYONCU, H. Exchange rate volatility and trade: An empirical investigation from cross-country comparison. African Development Review, v. 21, n. 3, p. 499-513, 1 dez. 2009.

SERENIS, D.; TSOUNIS, N. Exchange Rate Volatility and Aggregate Exports: Evidence from Two Small Countries. ISRN Economics, v. 2014, p. 1-10, 20 jan. 2014. 
SILVA, F. A.; FREITAS, C. O. DE; MATTOS, L. B. DE. Volatilidade da taxa de câmbio e seus efeitos sobre o fluxo de comércio dos países da América do Sul. Revista de Economia Contemporânea, v. 20, n. 2, p. 229-249, 2016.

TAKAENDESA, P. ; TSHEOLE, T. ; AZIAKPONO, M. Real exchange rate volatility and its effect on trade flows: new evidence from South Africa. Studies in Economics and Econometrics, v. 30, n. 3, p. 79-97, 1 nov. 2006.

TELES, V. K. Choques cambiais, política monetária e equilíbrio externo da economia brasileira em um ambiente de hysteresis. Economia Aplicada, v. 9, n. 55, p. 415-426, set. 2005.

VERGIL, H. Exchange Rate Volatility in Turkey and Its Effect on Trade Flows. Journal of Economic and Social Research, v. 4, n. 1, p. 83-99, 2002.

VITA, G. DE; ABBOTT, A. Real Exchange Rate Volatility and US Exports: An ARDL Bounds Testing Approach. Economic Issues Journal Articles, v. 9, n. 1, p. 69-78, 2004.

WANG, K.-L.; BARRETT, B. Estimating the Effects of Exchange Rate Volatility on Export Volumes. Journal of Agricultural and Resource Economics, v. 32, n. 2, p. 225-255, 2007.

WESSEH, P. K.; NIU, L. The impact of exchange rate volatility on trade flows: New evidence from South Africa. International Review of Business Research Papers, v. 8, n. 1, p. 140-165, 2012.

ZHANG, S.; BUONGIORNO, J. Effects of exchange rate volatility on export volume and prices of forest products. Canadian Journal of Forest Research, v. 40, n. 11, p. 2069-2081, nov. 2010. 